\title{
Intelligent Mobile Coronavirus Recognition Centre Based on IEEE 802.15.4
}

\author{
https://doi.org/10.3991/ijim.v15i16.24193 \\ Abdulkareem Alzahrani ${ }^{1(\mathbb{凶})}$, Khattab M Ali Alheeti², Samer Salah Thabit ${ }^{3}$, \\ Duaa Al Dosary ${ }^{2}$, Muzhir Shaban Al-Ani ${ }^{4}$ \\ ${ }^{1}$ Albaha University, Saudi Arabia \\ ${ }^{2}$ University of Anbar, Ramadi, Iraq \\ ${ }^{3}$ Almamoon University College, Baghdad, Iraq \\ ${ }^{4}$ University of Human Development, Iraq \\ ao.alzahrani@bu.edu.sa
}

\begin{abstract}
The novel coronavirus (COVID-19) has become widespread around the world. It started in Wuhan, China, and has since spread rapidly among people living in other countries. Hence, the World Health Organization has considered COVID-19 as a pandemic that threatens millions of people's lives. Due to the high number of infected people, many hospitals have been facing critical issues in providing the required medical services. For instance, some clinical centers have been unable to provide one of the most important medical services, namely blood tests to determine whether an individual is infected with COVID-19. Therefore, it is important to present an alternative diagnosis option to prevent the further spread of COVID-19. In this paper, a proposed intelligent detection communication system (IDCS) is configured for distributed mobile clinical centers to control the pandemic. In addition, the intelligent system is integrated with the Zigbee communication protocol to build a mobile COVID-19 detection system. The proposed system was trained on X-ray COVID-19 lung images used to identify infected people. The Zigbee protocol and decision tree algorithm were used to design the IDCS. The results of the proposed system show high accuracy $94.69 \%$ and accept results according to the performance measurements.
\end{abstract}

Keywords - artificial intelligence, COVID-19, decision tree algorithm, detection system

\section{$1 \quad$ Introduction}

The novel coronavirus (COVID-19) (shown in Figure 1) is a new strain of the coronavirus family first recorded in December 2019 in Wuhan, a large city in China. COVID-19 is one of a large group of viruses that can cause critical diseases (e.g. Middle East Respiratory Syndrome (MERS) and Severe Acute Respiratory Syndrome (SARS)) or common cold cases [1] [2]. The coronavirus infects humans and animals using similar techniques and can be rapidly spread from person to person when there is close contact with no protection. 


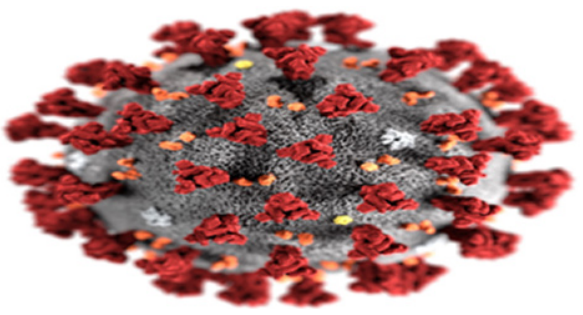

Fig. 1. Coronavirus (COVID-19) [3]

A large amount of COVID-19-related data can be generated within a few minutes, as the COVID-19 pandemic is happening in a highly connected and digitized world [4]. Artificial intelligence (AI) has shown great potential in predicting or tracing the source of infectious diseases. Therefore, it is essential to exploit the capabilities of AI and big data in the healthcare field [5].

The medical diagnosis of COVID-19 can be done through various techniques including a radiologist's diagnosis. Electronic Health Records (EHR) are used to count each certain new case each day and calculate the total sum of new cases [6]. In addition, thermal images or sensors gather immediate data to explore COVID-19 cases [1] [7].

Chest X-ray (CXR) radiographs and computed tomography (CT) scans involve a radiologist's diagnosis [8]. CXR or CT images are used to efficiently detect COVID19 symptoms. During recovery, chest CT scans can identify COVID-19 from the other types of pneumonia. Moreover, the scans can identify the stage of the patient's recovery or deterioration. By using 1,119 CT images, Artificial Neural Networks are utilized for healthy persons or infected with COVID-19 classification [9].

CT imaging is preferred for diagnosing pneumonia in COVID-19. Diagnoses by expert radiologists have shown accuracy identical to that obtained with a deep learning model [9]. Deep learning models can greatly enhance radiologists' performance in clinical practice. Therefore, deep learning can improve early diagnosis, treatment, and isolation and holds great potential to minimize the effort required from radiologists and control the pandemic [10]. Deep learning models can also be used to identify lesions in CT images and detect COVID-19 patients. These AI techniques enable rapid patient identification, achieving high performance on both classification tasks and pneumonia detection [11]. Using the feature pyramid network approach, feature extraction produces $86.7 \%$ accuracy using CT scans.

In this paper, a new mobile detection system is proposed for the early identification of infected people. It is composed of two main parts: a distributed communication system and a detection model. The Zigbee protocol (IEEE 802.15.4) is used to design the mobile wireless communication system. This system is configured at the main detention center, which receives inquiries from various medical points. This wireless network can provide security and ensure the privacy of patients' information. AI is used in the design of the detection system. More specifically, a decision tree algorithm is used to distinguish between normal and infected cases.

The main contributions of this paper are as follows:

- A communication system based heavily on the Zigbee protocol is proposed.

- A decision tree algorithm is used for the detection. 
The proposed system works by integrating Zigbee technology with a decision tree algorithm to detect COVID-19 infection versus non-infection.

The remainder of this paper is structured as follows. Section 2 presents related work. Section 3 presents the methodology of the proposed system. The proposed detection system is further explained in section 4. The testing of the proposed system and performance metrics are presented in section 5. Section 6 verifies the efficiency of the algorithm through simulation experiments. Finally, section 7 presents conclusions and suggests directions for future research.

\section{Related work}

With the COVID-19 pandemic on everyone's mind, many in society are considering how they can help. Many papers explore the use of AI in controlling the pandemic and fighting COVID-19; a selection of which are reviewed below.

The authors in [12] used deep learning for antivirals prediction, which could be used to assist coronavirus patients. Coronavirus RNA sequences can be used together with the model with the chemical combination to predict the best drug. The results of this research indicated that vidarabine, adenosine, and other compounds held promise.

The authors in [13] applied different models to research commercially obtainable drugs. In this research, the authors utilized a network called the molecular interaction transformer for drug-target interaction prediction. The authors fine-tuned this pre-trained model to predict 'binding affinity values between commercially available antiviral drugs and target proteins' and found that 'the 2019-nCoV 3C-like proteinase was predicted to bind with atazanavir'. This provides a good use case for adopting new deep learning architectures to different domains.

A system was proposed in [14] based on three different convolutional neural networks (CNN): InceptionV3, ResNet50, and InceptionResNetV2. The authors used CXR images to detect patients infected with coronavirus-related pneumonia. Their study presents insights into how deep learning methods can be used to detect COVID-19 at an early stage. They believed that if the proposed system demonstrated high performance, it could be used to help make decisions in clinical practice. The results showed that, among the three models, the proposed system demonstrated the highest accuracy (98\%) with the pre-trained ResNet50 model.

In [15], the authors used XGBoost to examine whether a COVID-19 patient would survive the infection based on many risk factors, such as age. This helps offer information about who should isolate themselves to avoid contracting the disease.

Santosh introduced and discussed the importance of training and testing models and using AI-driven tools to detect COVID-19 [16]. That research aimed to show that AI-driven tools are expected to engage in active learning through being trained and tested on several data types that are expected to be employed. The research discussed the use of multitudinal and multimodal data for active learning.

In [10], CT scans were classified using UNet++ to extract relevant features. The authors used 106 admitted patients to extract 40,000 scans for training. The model demonstrated high precision and recall. The proposed approach was highly efficient at optimizing radiologists' work. This exemplifies the positive effects of AI in real-world cases where radiologists can explore more CTs. 
$\mathrm{Xu}$ et al. collected information across three hospitals in China about 509 patients involving 175 healthy cases [17]. Their model showed low precision and recall, as they used fewer scans to train the model despite having more patients.

In [18], the authors used CT scans of the chest and automated AI tools for CT image analysis to monitor, quantify and detect COVID-19 cases and distinguish infected patients.

In [19], Apriori and decision tree algorithms were used to find similarities and differences between many amino acid sequences of MERS patients. After converting the DNA sequences of MERS with coronavirus into amino acid sequences, decision trees and Apriori algorithms were used.

Through searching and analyzing the literature, we developed the previously mentioned proposed system. Our paper is distinguished from others by presenting a CXRbased COVID-19 detection system using the Zigbee protocol and a decision tree algorithm. The study's motivation is the detection of patients infected with COVID-19 using CXRs. The proposed method uses deep features to distinguish COVID-19 CXR images from non-affected CXR images.

\section{$3 \quad$ Methodology of the detection system}

In this paper, the proposed system is implemented as shown in Figure 2. First, data are collected from patients at various medical points, then transmitted to the nearest hospital. Second, the receiver is connected to a PC at the hospital to receive the transmitted signals to allow for quick hospital intervention. Zigbee-based wireless technology is used for wireless data transmission. A detailed description of the system components is given in the following section.

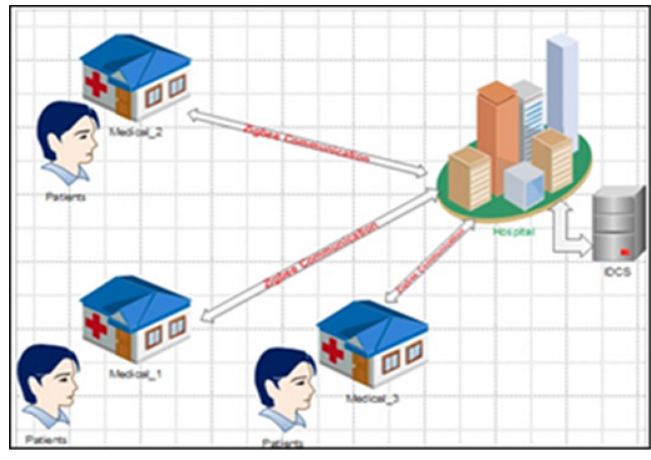

Fig. 2. Data acquisition and transmission

In this scheme, normal CXRs are collected at various medical points for people infected with COVID-19 as well as non-infected people. These images are then sent to the closest detection system. Zigbee communication plays an important role in transmitting CXR images from medical points as well as receiving these images at a hospital that includes an intelligent detection communication system (IDCS). It also sends the detection result from the IDCS to inquire centre. The dataset is divided into three subsets: training, validation, and testing. A decision tree machine learning algorithm was applied for classification. 


\subsection{Zigbee technology}

Zigbee is a wireless communication technology based on IEEE 802.15.4 with low cost, low power consumption, small size, and low rate of transmission. It is a high-level communication protocol designed to generate personal area networks for low-bandwidth needs, such as home automation and medical device data collection [20]. Features of Zigbee include the following [21]:

a) The module cost of the Zigbee network is low, and the code is open source.

b) Its power consumption is low.

c) It reduces competition and conflicts when data is transmitted and, if there is a problem, it supports retransmission.

d) It has a large network capacity.

e) Zigbee supports authentication and packet integrity checking.

The architecture of the Zigbee protocol is presented in Figure 3.

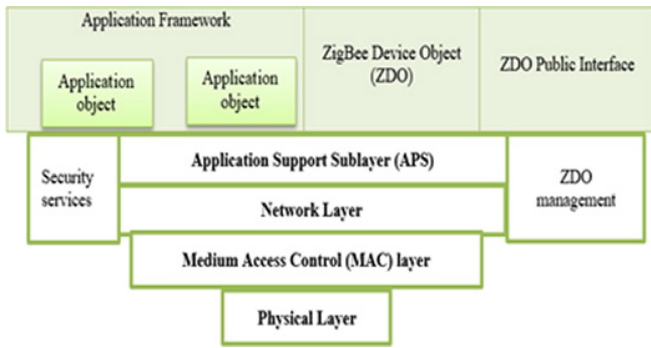

Fig. 3. Zigbee architecture

The network layer is responsible for route discovery, neighbor discovery, Zigbee Device Object (ZDO) management, and security. The ZDO controls and manages application objects, initializes the coordinator, and provides security services.

\subsection{Decision trees}

Decision trees, also known as reduction trees or classification trees [22], are a supervised machine learning technique that produces a decision tree from training data. A decision tree is a predictive model that concludes the target value based on observations about an item. In the structures of the decision tree, no-leaf nodes represent features, leaves or labels represent classifications and branches represent conjunctions of features that point to the classifications.

A decision tree algorithm can be used to solve classification and regression problems. To predict the value of target variables or classes, a decision tree is used to learn decision rules inferred from training data. Identifying which attributes are needed at the root node and each subsequent level is the main challenge in decision tree implementation. Decision trees are a good choice for understanding and making good interpretations of data because they mimic human thought. 


\subsection{Dataset description}

In this paper, CXR images were gathered from healthy (i.e., non-infected) patients as well as patients who had tested positive for COVID-19. Twenty CXRs of normal lungs were taken from the Shenzhen Hospital X-ray Set and 30 CXRs of COVID-19 patient lungs from X-ray-dataset-master, as well as 15 CXRs of COVID-19 patient lungs from $\mathrm{x}$-ray-dataset-master and 15 normal CXRs from the Shenzhen Hospital X-ray Set [23]. Table 1 shows some of the images utilised in this paper.

Table 1. Examples from a dataset of X-ray images in normal cases and COVID-19 patients

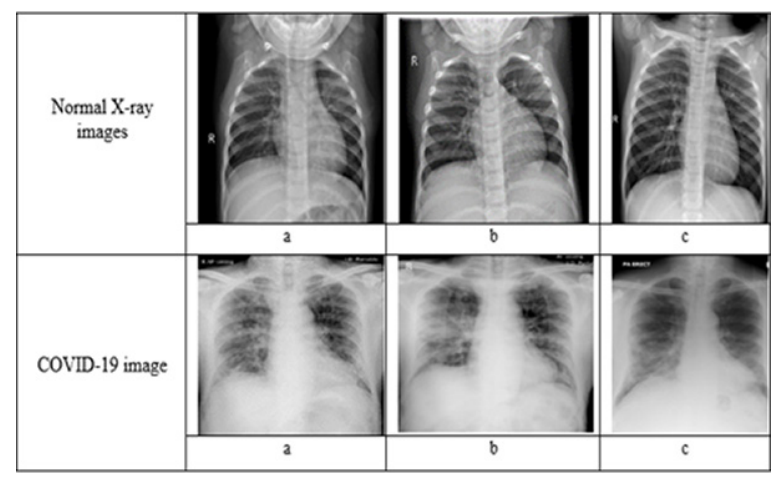

\subsection{Simulation environment}

Two files were generated from the simulation in Network Simulator version 2 (ns-2): the trace file (text) and NAM file. The trace file generated in ns- 2 is employed in an extracted dataset, whereas the NAM file is utilized to present a connection environment of nodes (medical points), as shown in Figure 4.

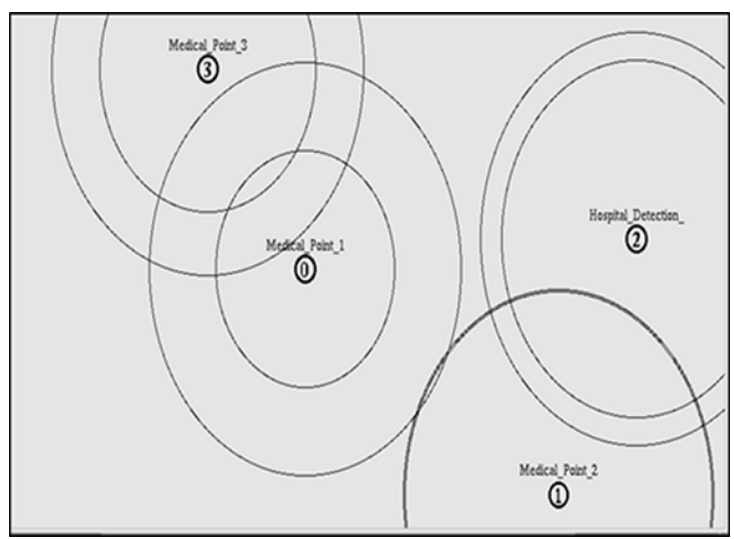

Fig. 4. Screenshot of simulation of NAM file in ns2 


\section{$4 \quad$ Proposed detection system}

Non-infected (normal) CXRs from healthy patients were collected along with CXRs of patients who had tested positive for COVID-19. Ns-2 was used to generate communications between medical points. The output of the simulation generated by ns-2 is composed of two files: the NAM and trace (text) files. The NAM file is used to present a connection environment of nodes, whereas the trace file is employed to an extracted dataset. Pre-processing is needed for data extracted from the trace file to convert all symbols and characters into numeric values. The dataset is then divided into training, validation, and testing sets. Decision tree machine learning was then applied to distinguish infected from non-infected lungs. The overall architecture of the proposed system is presented in Figure 5.

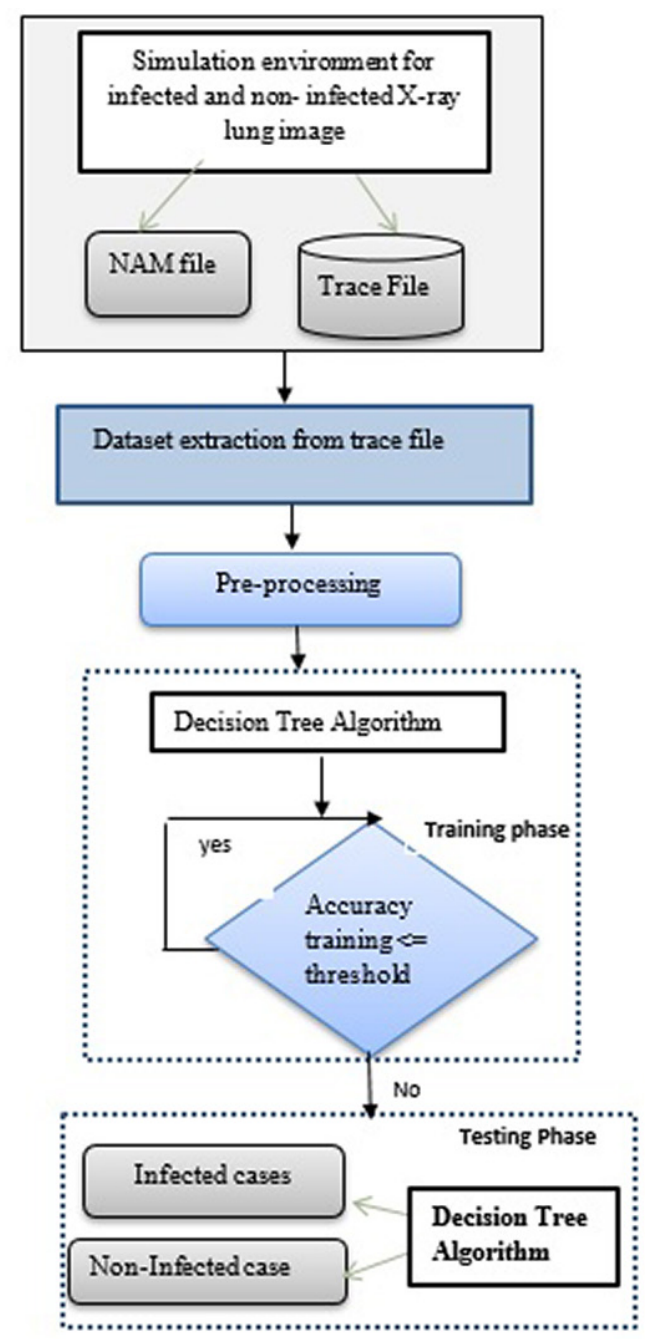

Fig. 5. A proposed COVID-19 detection system 


\section{$5 \quad$ Performance measurement}

Two types of performance metrics were used to measure the efficiency of the proposed system.

\subsection{Detection system}

Our proposed system is composed of two parts: a detection system and a communication system. The metrics of accuracy and confusion matrix were calculated as follows [24]:

$$
\text { Accuracy }=\frac{\text { Number of correctly classified patterns }}{\text { Total number of patterns }}
$$

To measure and evaluate the proposed system's performance, it is necessary to calculate four rates: true positive (TP), false positive (FP), true negative (TN), and false negative (FN). These measures are calculated as follows [25]:

$$
\begin{aligned}
\mathrm{TP}_{\text {Rate(sensitivity) }} & =\frac{\mathrm{TP}}{\mathrm{TP}+\mathrm{FP}} \\
\mathrm{TN}_{\text {Rate(specificity) }} & =\frac{\mathrm{TN}}{\mathrm{TN}+\mathrm{FP}} \\
\mathrm{FN}_{\text {Rate(1-sensitivity) }} & =\frac{\mathrm{FN}}{\mathrm{FN}+\mathrm{TP}} \\
\mathrm{FP}_{\text {Rate(1-specificity) }} & =\frac{\mathrm{FP}}{\mathrm{FP}+\mathrm{TN}}
\end{aligned}
$$

a) TP: the number of correctly predicted COVID-19 cases. TP is also known as Recall.

b) FP: the number of incorrectly predicted COVID-19 cases, where positive represents the total number of COVID-19 cases.

c) TN: the number of correctly predicted non-COVID-19 cases.

d) FN: the number of incorrectly predicted non-COVID-19 cases, where negative represents the total number of non-COVID-19 cases.

Precision, the last metric used in this paper, was calculated as follows:

$$
\text { Precision }=\frac{\mathrm{TP}}{\mathrm{TP}+\mathrm{FP}}
$$




\subsection{Communication system}

Other performance metrics were employed to calculate the efficiency of the proposed communication system after integrating it with a detection system. These metrics include packet delivery rate (PDR), throughput, and end-to-end delay [26].

$$
P D R=\sum N_{r} / \sum N_{s}
$$

where $N_{r}$ is the number of packets received and $N_{s}$ is the number of packets sent.

$$
\text { Throughput }(\mathrm{kbp})=\frac{N_{r} \times S}{S T}
$$

where $N_{r}$ is the number of packets received, $S$ is the packet size and $S$ is the simulation time?

$$
\text { End }- \text { to }- \text { End Delay }(\mathrm{ms})=\left(\frac{\sum \text { end }_{\text {time }}-\text { start }_{\text {time }}}{\sum N}\right)
$$

where $N$ represents several connections.

\section{Experimental results}

The intelligent detection system proposed in this paper was implemented in Python. The Zigbee communication system integrated with the detection system is configured with ns-2.

In the experiments, the system's performance concerning COVID-19 identification was examined. Decision tree machine learning was applied to distinguish infected from non-infected lungs. The results for the confusion matrix, detection rate, accuracy, precision, and recall are illustrated in Table 2, Table 3, and Table 4.

Table 2. Confusion matrix

\begin{tabular}{|c|c|c|c|}
\hline TP & TN & FN & FP \\
\hline $97.32 \%$ & $97.15 \%$ & $2.68 \%$ & $2.85 \%$ \\
\hline
\end{tabular}

These rates represent the confusion matrix for the detection system.

Table 3. Detection rates

\begin{tabular}{|c|c|c|c|c|c|}
\hline Original & Matching & Normal Cases & Original & Matching & COVID-19 Cases \\
\hline 763 & 759 & $99.47 \%$ & 610 & 609 & $99.83 \%$ \\
\hline
\end{tabular}

Table 3 indicates the efficiency of the IDCS in terms of detection rate. 
Table 4. Performance metrics

\begin{tabular}{|c|c|c|}
\hline Precision & Recall & Accuracy \\
\hline $97.22 \%$ & $97.32 \%$ & $94.69 \%$ \\
\hline
\end{tabular}

The performance metrics provided in Table 4 are reflected in the successful application of the IDCS to reduce the number of infected people. Throughput, PDR, and end-to-end delay are presented in Table 5.

Table 5. Performance metrics of communication system

\begin{tabular}{|c|c|c|}
\hline Throughput & PDR & End-to-End Delay \\
\hline $11.56 \mathrm{kbps}$ & $81.2 \%$ & $14.15 \mathrm{~ms}$ \\
\hline
\end{tabular}

Table 5 shows the significant performance metrics: throughput, PDR, and end-toend delay of the IDCS system.

\section{Conclusions and future directions}

This research aims to prove the classification model's performance in COVID-19 identification. A proposed system was introduced to detect whether people are or are not infected with COVID-19 and then send their cases to the nearest hospital in the patient area using the Zigbee protocol. In this paper, we presented a detection system that could distinguish an infected patient with COVID-19 from others using a decision tree algorithm and Zigbee technology. The system performance was verified via the performance metrics. A confusion matrix was also computed. The simulation results demonstrated an overall accuracy rate of $94.69 \%$ and could be a helpful diagnostic tool for doctors. In future research, the detection system should incorporate other AI tools, such as linear discrimination analysis, support vector machines and fuzzy Petri nets. In addition, this system could be integrated with other health systems.

\section{References}

[1] Elmousalami H. H. and A. E. Hassanien, "Day Level Forecasting for Coronavirus Disease (COVID-19) Spread: Analysis, Modeling and Recommendations," 2020.

[2] Memish Z. A., S. Perlman, M. D. Van Kerkhove, and A. Zumla, "Middle East respiratory syndrome," Lancet, vol. 395, no. 10229, pp. 1063-1077, 2020. https://doi.org/10.1016/ $\underline{\text { S0140-6736(19)33221-0 }}$

[3] "FDA approves emergency use of coronavirus diagnostic test." [Online]. Available: https:// www.medicaldevice-network.com/news/fda-coronavirus-test/. [Accessed: 26-Apr-2020].

[4] Tuaycharoen, Nuengwong. "University-Wide Online Learning During COVID-19: From Policy to Practice.” (2021): 38-54 https://doi.org/10.3991/ijim.v15i02.18143

[5] Anthony O., “A Propose Machine Learning approach for Monitoring Individual's Health Status on Corona virus (COVID19) cases," Academia.edu, no. March, p. 1, 2020.

[6] F. H. • L. K.・J. Vanschoren, Automated Machine Learning. Springer, 2019.

[7] Allam Z. and D. Jones, "On the Coronavirus (COVID-19) Outbreak and the Smart City Network: Universal Data Sharing Standards Coupled with Artificial Intelligence (AI) to Benefit Urban Health Monitoring and Management," Healthcare, vol. 8, 2020. https://doi. org $/ 10.3390 /$ healthcare 8010046 
[8] Molnar C., Interpretable machine learning. Lulu. com, 2019.

[9] Shuai B. X. et al., "A deep learning algorithm using CT images to screen for Corona Virus Disease (COVID-19)," 2020.

[10] Jun H. Y. Chen et al., "Deep learning-based model for detecting 2019 novel coronavirus pneumonia on high-resolution computed tomography: a prospective study," 2020.

[11] Song Y. et al., "Deep learning Enables Accurate Diagnosis of Novel Coronavirus (COVID19) with CT images," medRxiv, 2020. https://doi.org/10.1109/TCBB.2021.3065361

[12] Haiping M. T. H. Zhang et al., "Deep learning-based drug screening for novel coronavirus 2019-nCov," Prepr., vol. 19, no. 8, pp. 1-17, 2020.

[13] Beck B. R., B. Shin, Y. Choi, S. Park, and K. Kang, "Predicting commercially available antiviral drugs that may act on the novel coronavirus (2019-nCoV), Wuhan, China through a drug-target interaction deep learning model," bioRxiv, 2020. https://doi.org/10.1101/2020.01.31.929547

[14] Ali Z. P. Narin, Ceren Kaya, "Automatic Detection of Coronavirus Disease (COVID19) Using X-ray Images and Deep Convolutional Neural Networks," 2020. https://doi. org/10.1007/s10044-021-00984-y

[15] Yan L. et al., "Prediction of criticality in patients with severe Covid-19 infection using three clinical features: a machine learning-based prognostic model with clinical data in Wuhan," medRxiv, 2020.

[16] Santosh K. C., "AI-Driven Tools for Coronavirus Outbreak: Need of Active Learning and Cross-Population Train/Test Models on Multitudinal/Multimodal Data," J. Med. Syst., vol. 44, no. 5, pp. 1-5, 2020. https://doi.org/10.1007/s10916-020-01562-1

[17] Xu X. et al., "Deep Learning System to Screen Coronavirus Disease 2019 Pneumonia," pp. 1-29, 2020. https://doi.org/10.1016/j.eng.2020.04.010

[18] Gozes O., M. Frid-Adar, H. Greenspan, P. D. Browning, A. Bernheim, and E. Siegel, "Rapid AI Development Cycle for the Coronavirus (COVID-19) Pandemic: Initial Results for Automated Detection \& Patient Monitoring using Deep Learning CT Image Analysis,” 2020.

[19] Yoon T. et al., "Analysis of transmission route of MERS coronavirus using decision tree and Apriori algorithm," 2016 18th Int. Conf. Adv. Commun. Technol., pp. 559-565, 2016.

[20]“Zigbee.” [Online]. Available: https://en.wikipedia.org/wiki/Zigbee. [Accessed: 12-Apr-2020].

[21] Tang Y., H. Lian, L. Li, X. Wang, and X. Yan, "A randomness detection method of zigbee protocol in a wireless sensor network," Sensors (Switzerland), vol. 18, no. 11, pp. 1-11, 2018. https://doi.org/10.3390/s18113962

[22] Tan L., "Code Comment Analysis for Improving Software Quality." [Online]. Available: https://www.sciencedirect.com/topics/computer-science/decision-trees. [Accessed: 09-Apr-2020].

[23] "No Title." [Online]. Available: https://github.com/ieee8023/covid-chestxray-dataset. [Accessed: 25-Apr-2020].

[24] Alheeti K. M. Ali et al., "The affect of fuzzification on neural networks intrusion detection system," 2009 4th IEEE Conf. Ind. Electron. Appl. ICIEA 2009, pp. 1236-1241, 2009.

[25] Hussein A. S., R. S. Khairy, S. M. M. Najeeb, and H. T. ALRikabi, "Credit Card Fraud Detection Using Fuzzy Rough Nearest Neighbor and Sequential Minimal Optimization with Logistic Regression," International Journal of Interactive Mobile Technologies, vol. 15, no. 5, 2021. https://doi.org/10.3991/ijim.v15i05.17173

[26] Sumaya H., Khattab M, Yossra H. and Shaimaa H. S., "Clustering and Analysis of Dynamic Ad Hoc Network Nodes Movement Based on FCM Algorithm", International Journal of Online and Biomedical Engineering, ISSN 18681646, 18612121, Vol. 16, No. 12, 2020. https://doi.org/10.3991/ijoe.v16i12.16067 


\section{Authors}

Abdulkareem Alzahrani received his bachelor's degree from the department of Computer Science at Abha Teacher Collage, Saudi Arabia (2007). He received MSc in Advanced Web Engineering from the University of Essex, UK (2011) and PhD in Computer Science from the same University (2017). He then works at the Faculty of Computer Science and Information Technology, Albaha University, Saudi Arabia as an assistant professor. His research fields are intelligent environment, soft computing and multi-agent systems.

Khattab M. Ali Alheeti received the B.Sc. Computer Science degree in 2000 from Baghdad, Iraq, M.Sc. (Hons.) Computer Networking and Information in 2009 from the University of Al Al-Bayt, Jordan, and Ph.D. from Essex University in 2017, respectively. Currently, he is working at University of Anbar.

Samer Salah Thabit was born in Baghdad, Iraq, in 1978. He received his B.S. degree in Department of Computer Science, AlMa'mon University College, Baghdad, Iraq, in 2001, the M.S degree in information system, Osmania University, India, in 2012, he is a Teacher at the Computer science Department, AlMa'mon University College. His research interests include mobile communication, cooperative communications and networking, cloud computing, features of network architecture.

Duaa Abdul Sattar Rukan, born in 1987, Iraq, Anbar, holds a master's degree in computer science from the College of Computer Science and Information Technology, Anbar University, my interests are in the field of networks and information security.

Prof. Dr. Muzhir Shaban Al-Ani has received Ph. D. in Computer \& Communication Engineering Technology, ETSII, Valladolid University, Spain, 1994. Assistant of Dean at Al-Anbar Technical Institute (1985). Head of Electrical Department at Al-Anbar Technical Institute, Iraq (1985-1988), Head of Computer and Software Engineering Department at Al-Mustansyria University, Iraq (1997-2001), Dean of Computer Science (CS) \& Information System (IS) faculty at University of Technology, Iraq (2001-2003). He joined in 15 September 2003 Electrical and Computer Engineering Department, College of Engineering, Applied Science University, Amman, Jordan, as Associated Professor. He joined in 15 September 2005 Management Information System Department, Amman Arab University, Amman, Jordan, as Associated Professor, then he joined computer science department in 15 September 2008 at the same university. He joined in 15 August 2009 College of Computer, Al-Anbar University, Iraq. He joined in 1 October 2016 Department of Computer Science - College of Science and Technology - University of Human Development - KRG - Iraq.

Article submitted 2021-05-22. Resubmitted 2021-06-27. Final acceptance 2021-06-28. Final version published as submitted by the authors. 